NASZA DERMATOLOGIA Online OUR DERMATOLOGY Online

Source of Support:

Georgia Dermatopathology

Associates, Atlanta, Georgia, USA

Competing Interests:

None

\section{A TRANSIENT DRUG INDUCED LUPUS ERYTHEMATOSUS- LIKE ALLERGIC DRUG REACTION WITH MULTIPLE ANTIBODIES}

\author{
Ana Maria Abreu Velez ${ }^{1}$, Vickie M. Brown², Michael S. Howard ${ }^{1}$ \\ ${ }^{1}$ Georgia Dermatopathology Associates, Atlanta, Georgia, USA \\ ${ }^{2}$ Family Dermatology, Milledgeville, Georgia, USA
}

Corresponding author: Ana Maria Abreu Velez, MD PhD

abreuvelez@yahoo.com

\begin{abstract}
Drug reactions may mimic several dermatoses, including lupus erythematosus. We present an 80 year old female patient on multiple medications, who presented with blisters on her hands and arms for two weeks, which then generalized to the rest of her body. The patient was evaluated by a dermatologist, and biopsies for hematoxylin and eosin (H\&E) examination, as well as for direct immunofluorescence (DIF) and immunohistochemistry (IHC) were performed. The H\&E biopsy examination revealed a mild, superficial, perivascular dermal infiltrate of lymphocytes, histiocytes and abundant eosinophils; neutrophils were rare. No vasculitis was noted. DIF revealed positive basement membrane (BMZ) staining, primarily with patchy Complement/C3c and fibrinogen; in addition, strong reactivity to dermal blood vessel was appreciated. Antibodies to cell junction-like structures were also noted in the epidermis and dermis with these two antibodies. IHC using similar immunoglobulins and complement components showed similar patterns. We observed that contrary to lupus erythematosus, neither IgG nor IgM were positive at the BMZ.
\end{abstract}

Key words: aspirin; lisinopril; Nexium; hydralazine; Tylenol

Abbreviation: Drug-induced lupus erythematosus (DIL), systemic lupus erythematosus (SLE), basement membrane zone (BMZ), direct immunofluorescence (DIF)

Cite this article:

Ana Maria Abreu Velez, Vickie M. Brown, Michael S. Howard: A transient drug induced lupus erythematosus - like allergic drug reaction with multiple antibodies. Our Dermatol Online. 2013; 4(4): 511-513

\section{Introduction}

Drug-induced lupus erythematosus (DIL) is an autoimmune disorder, caused by chronic use of selected medications. These drugs cause an autoimmune response that can produce a clinical presentation similar to those of systemic lupus erythematosus (SLE). There are multiple known medications that elicit DIL, but three are most strongly associated: hydralazine, procainamide, and isoniazid [1-3].

\section{Case Report}

We present an 80 year old female patient, who was using multiple medications and presented blisters on her hands and arms for two weeks. With the rash, the patient described flulike symptoms. The patient also had a history of chronic leg lymphedema. Physical exam displayed erythematous papules and wheals, with no hyperpigmentation or atrophy. The patient was taking oral simvastatin, aspirin $81 \mathrm{mgs} /$ day, lisinopril, Januvia ${ }^{\circledR}$, Nexium ${ }^{\circledR}$, hydralazine, Tylenol, and metropolol tartrate. On physical exam, the lesions were present on the bilateral forearms, hands and wrists, and focally on the face and legs. Skin biopsies for hematoxylin and eosin (H\&E) examination, as well as for direct immunofluorescence (DIF) and immunohistochemistry (IHC) were performed and processed as previously described [4-7].

\section{Microscopic examination:}

Examination of the H\&E tissue sections demonstrated a histologically unremarkable epidermis. No subepidermal blistering was noted. Within the dermis, a mild, superficial, perivascular infiltrate of lymphocytes, histiocytes and abundant eosinophils was identified. Neutrophils were rare (Fig. 1).

\section{Direct immunofluorescence (DIF):}

DIF was performed, and revealed the following results: $\operatorname{IgE}$ (+, focal superficial dermal perivascular); Complement/C1q $(-)$; complement/C3c (+++, shaggy in patches at the basement membrane zone (BMZ), in the dermal blood vessels and directed against some types of cell junctions in the epidermis and the dermis); Complement/C4(+, focal dermal cell junctions); and fibrinogen(patterns and positivity similar to C3c) (Fig. 1). 
Fibrinogen and kappa light chain antibodies stained focal subcorneal areas. Epidermal cytoid bodies were observed, and demonstrated positive staining with $\operatorname{IgM}$, fibrinogen, kappa light chains and complement/C3c. Antibodies to Ro/SSA-were positive, predominately with Complement/C3c. Anti-keratin antibodies were seen in the epidermis with IgM (Fig. 2).

Immunostochemistry staining revealed similar findings to those seen by DIF, and also reactivity to dermal blood vessels with IgA (Fig. 1).

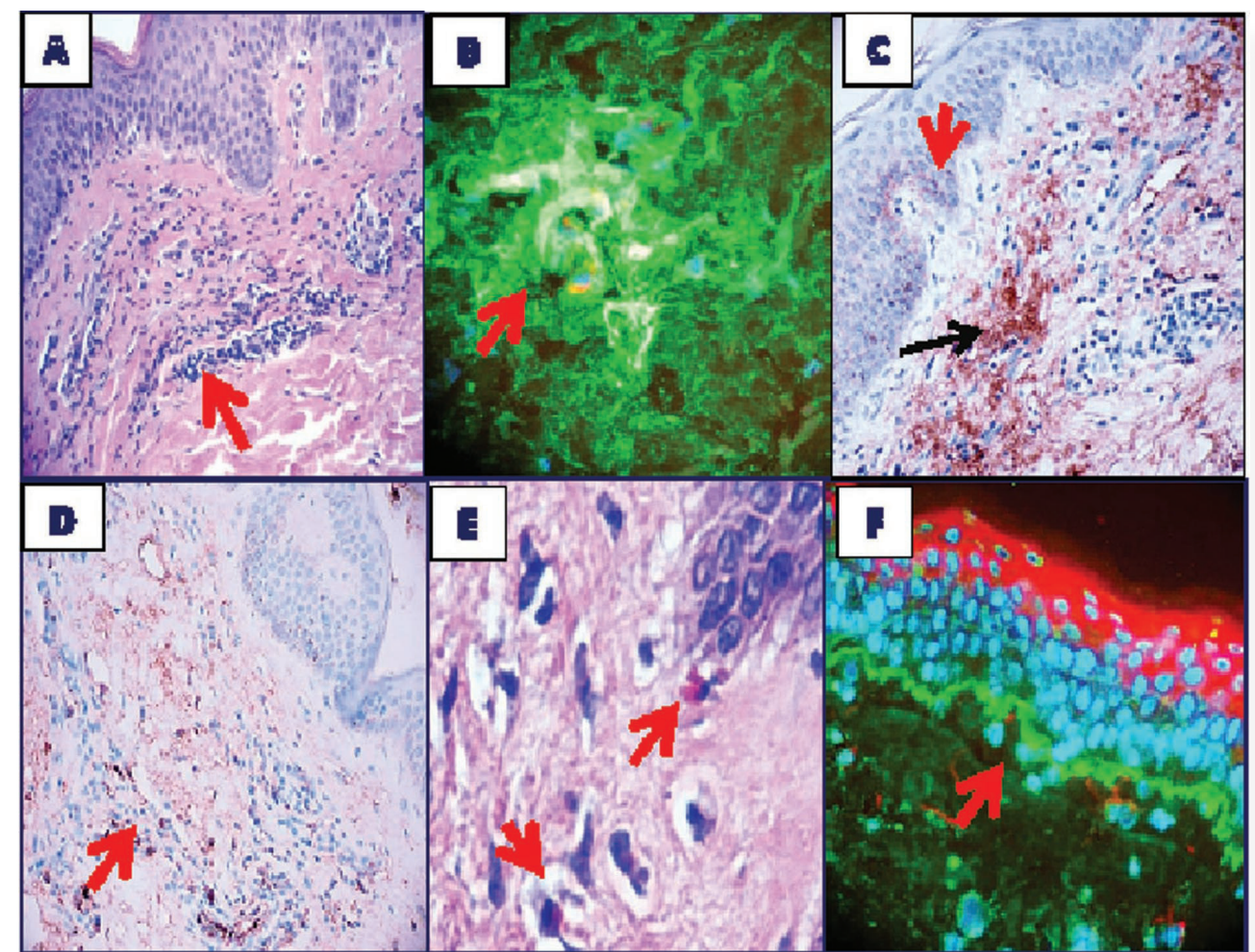

Figure 1. a. H\&E staining demonstrates a mild, superficial, perivascular dermal infiltrate (red arrow). b. DIF, demonstrating positive staining around dermal blood vessels with FITC conjugated fibrinogen(yellow staining; red arrow). c. Positive IHC staining with anti-human Complement/C3c in patches at the basement membrane zone (brown staining; red arrow) as well as around upper dermal blood vessels (brown staining; black arrow). d. IHC positive staining with anti-human IgA, around upper dermal blood vessels (brown staining; red arrow). e. H\&E stain, demonstrating dermal infiltrate eosinophils(red arrows)(400X) $\mathrm{f}$. DIF, demonstrating positive staining at the basement membrane zone with FITC conjugated C3c (yellow/green staining; red arrow). Epidermal keratinocyte nuclei were counterstained with Dapi (blue), and the upper layers of the epidermis were stained with rhodamine conjugated Ulex europaeus agglutinin(pink/red staining).

\section{Discussion}

Our patient was taking many medications that have been associated with DIL, and thus this diagnosis was favored given our pathologic findings. Clinically, DIL patients often report flu-like and joint discomfort symptoms. Our patient reported a chronic osteoarthalgia, and we could not determine if our DIL diagnosis contributed to this clinical problem. Additional signs and symptoms of DIL include myalgia, fatigue, pericarditis and/ or pleuritis; in addition, positive anti-histone antibodies are noted in $95 \%$ of cases [1-3]. Our patient was negative for antihistone antibodies, but positive for antibodies against SS-A/Ro with Complement/C3c.

In DIL, the lesions classically recede after discontinuing use of the eliciting drugs [1-3]. For therapy of our patient, Tacrolimus ${ }^{\circledR}$ $1 \%$ cream was prescribed 3 times a day topically, as well as triamcinolone acetonide $0.1 \%$ topical cream. In recalcitrant cases of DIL, it may be also necessary to add systemic corticosteroids and/other immunosuppressive agents [3].

Similar to expected findings in lupus erythematosus, we observed cytoid bodies with IgM antibodies. In addition, in our case we noted cytoid body antibodies with Complement/C3c, fibrinogen and subcorneal antibodies that are not classically described in lupus. We observed tissue fixed deposits of immunoglobulin M present in the cytoplasm of epidermal keratynocytes; however, instead of the 3 reported patters described in normal skin $[8,9]$, our reactivity was present throughout the entire epidermis. Also remarkable was the reactivity to some type(s) of likely cell junctions in the epidermis and dermis, best appreciated with anti-Complment/C $3 \mathrm{c}$ and fibrinogen.

In conclusion, we note differences with classic lupus erythematosus in our case, including lack of an H\&E interface dermatitis and the H\&E presence of dermal eosinophils. Our case also features immunopathological features that differ from lupus; we noted positive antibodies to Ro/SSA, but with Complement/C3c. Further, in DIF and IHC our case differs from classic lupus due to a lack of linear deposits of $\operatorname{IgG}$ or IgM at the basement membrane zone, instead, patchy Complement/ $\mathrm{C} 3 \mathrm{c}$ and fibrinogen were observed. We also observed significant reactivity to dermal blood vessels, but no vasculitis as noted in selected classic cases of lupus. We also observed reactivity to several likely cell junctions in epidermis and dermis, whose nature remains unknown. Further studies utilizing sera against epidermal and dermal antigens may be helpful in characterizing these putative epitopes. 

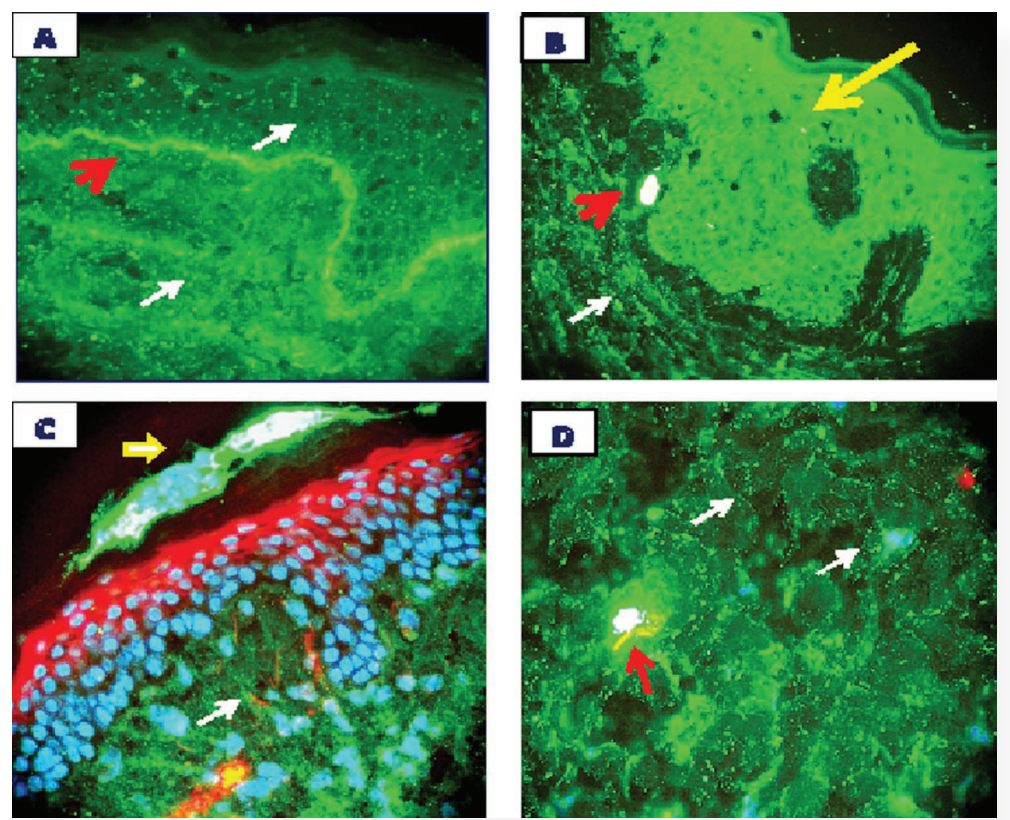

Figure 2. a. DIF, demonstrating positive staining at the basement membrane zone with FITC conjugated Complement/C3c (yellow staining; red arrow); also note the multiple cell junction-like staining, visualized as small dots in the dermis and epidermis (yellow staining; white arrows). b. DIF, with positive staining against cytoid bodies with FITC conjugated anti-human IgM (yellow staining; red arrow). Also noted additional staining with this antibody as anti-keratin antibodies in the epidermis (ie, cytoplasmic antigens) (yellow staining; yellow arrow), as well as to some type of cell junction-like structures in the dermis (yellow staining; white arrow). c. DIF, showing positive staining within the epidermal stratum corneum with FITC conjugated Complement/C3c(yellow staining; yellow arrow). Epidermal keratinocyte nuclei were counterstained with Dapi (blue), and the upper layers of the epidermis were stained with rhodamine conjugated Ulex europaeus agglutinin. The white arrow highlights additional positive, focal staining against cell junction-like structures in the dermis (yellow staining). d. DIF, displaying positive staining with FITC conjugated antihuman fibrinogen, in a dermal perivascular distribution (yellow staining; red arrow); in addition, note the punctuate positive staining against cell junction-like structures in the dermis (yellow staining; white arrows).

\section{REFERENCES}

1. Hofstra A: Metabolism of hydralazine: relevance to drug-induced lupus. Drug Metab Rev. 1994.26:485-505.

2. Marzano AV, Vezzoli P, Crosti C: Drug-induced lupus: an update on its dermatologic aspects. Lupus. 2009;18:935-40.

3. Sarzi-Puttini P, Atzeni F, Capsoni F, Lubrano E, Doria A: Druginduced lupus erythematosus. Autoimmunity. 2005;38:507-18.

4. Abreu Velez, AM, Klein AD, Howard MS: Specific cutaneous histologic and Immunologic features in a case of early lupus erythematosus scarring alopecia Our Dermatol Online. 2013;4:199201.

5. Abreu Velez, AM, Klein AD, Howard MS: Jam-A, plasminogen and fibrinogen Reactivity in a case of a lupus erythematosus-Like allergic drug reaction to Lisinopril. J Clin Exp Dermatol Res. 2012;S:6.
6. Abreu-Velez AM, Klein AD, Howard MS: Skin appendageal immune reactivity in a case of cutaneous lupus. Our Dermatol Online. 2011; 2:175-80.

7. Abreu-Velez AM, Smith JG Jr, Howard MS: Activation of the signaling cascade in response to $\mathrm{T}$ lymphocyte receptor stimulation and prostanoids in a case of cutaneous lupus. North Am J Med Sci. 2011;3:251-4.

8. Ioannides D, Bystryn JC: Association of tissue-fixed cytoplasmic deposits of immunoglobulin in epidermal keratinocytes with lupus erythematosus. Arch Dermatol. 1993;129:1130-25.

9. Bystryn JC, Nash M, Robins P: Epidermal cytoplasmic antigens: II. Concurrent presence of antigens of different specificities in normal human skin. J Invest Dermatol. 1978;71:110-3.

Copyright by Ana Maria Abreu Velez, et al. This is an open access article distributed under the terms of the Creative Commons Attribution License, which permits unrestricted use, distribution, and reproduction in any medium, provided the original author and source are credited. 